17 | 2021

René Maran

\title{
René Maran - Mohammed Dib
}

D'un passage de témoin

Hervé Sanson

\section{(2) OpenEdition}

1 Journals

Édition électronique

URL : https://journals.openedition.org/coma/7088

DOI : 10.4000/coma.7088

ISSN : 2275-1742

\section{Éditeur}

Institut des textes \& manuscrits modernes (ITEM)

\section{Référence électronique}

Hervé Sanson, « René Maran - Mohammed Dib », Continents manuscrits [En ligne], 17 | 2021, mis en ligne le 15 octobre 2021, consulté le 12 janvier 2023. URL : http://journals.openedition.org/coma/7088 ; DOI : https://doi.org/10.4000/coma.7088

Ce document a été généré automatiquement le 12 janvier 2023.

\section{(c) (i) $\ominus$}

Creative Commons - Attribution - Pas d'Utilisation Commerciale - Pas de Modification 4.0 International - CC BY-NC-ND 4.0

https://creativecommons.org/licenses/by-nc-nd/4.0/ 


\title{
René Maran - Mohammed Dib
}

\author{
D'un passage de témoin
}

Hervé Sanson
«Surtout, ne me parlez ni de la France, ni des Français et de leur esprit fraternel ! La fraternité n'est qu'un mot. S'il a quelque valeur en France, pour les Français de France, ceux des colonies ne lui en reconnaissent aucune ${ }^{1}$. »

1 René Maran et Mohammed Dib ne se sont jamais fréquentés, ni même rencontrés. Une génération les sépare, outre leur appartenance à un champ géographique différent. Maran né en 1883 en Martinique de parents guyanais, ayant fait ses études à Bordeaux, ayant ensuite exercé en tant qu'administrateur colonial durant une douzaine d'années au Congo-Brazza ainsi qu'en Oubangui-Chari avant de rentrer en France, et Mohammed Dib né en 1920, en Algérie coloniale, à Tlemcen dans l'Ouest algérien, installé en France à partir de 1960, ont tous deux intégré le champ dit francophone, trop souvent occulté, ou a minima marginalisé, dans le « centre » culturel qu'est la France métropolitaine.

2 Maran a pourtant consacré une note de lecture fort intéressante au premier roman de Mohammed Dib. L'auteur de Batouala a publié avant et après-guerre un certain nombre de recensions critiques dans la presse de son époque : La Dépêche de Toulouse, La Corrèze républicaine, Les Lettres françaises, Les Belles Lectures, Le Journal du Peuple, Gavroche, ou bien encore Présence africaine sont quelques-uns des périodiques ayant accueilli en leurs colonnes ses contributions. Ses notes de lecture sont essentiellement consacrées aux littératures «noires »: que ce soit celles afro-américaines, les littératures antillaises francophone, anglophone, ou hispanophone, mais aussi les littératures d'Afrique. Sa recension consacrée à La Grande Maison, roman paru au printemps 1952, publiée dans La Corrèze républicaine le 20 juin 1952, apparaît donc exceptionnelle. Cette année, paraissaient deux romans fondateurs de la littérature francophone d'Afrique du Nord: le roman de Dib, mais aussi le premier roman de Mouloud Mammeri, La Colline oubliée. Qu'a pu déceler Maran d'unique dans le roman de l'écrivain algérien ? Celui-ci lui a-t-il permis de formuler certains paramètres inhérents à sa vision idéologique et politique? 
Fig. 1 : Recension de René Maran de La Grande Maison.

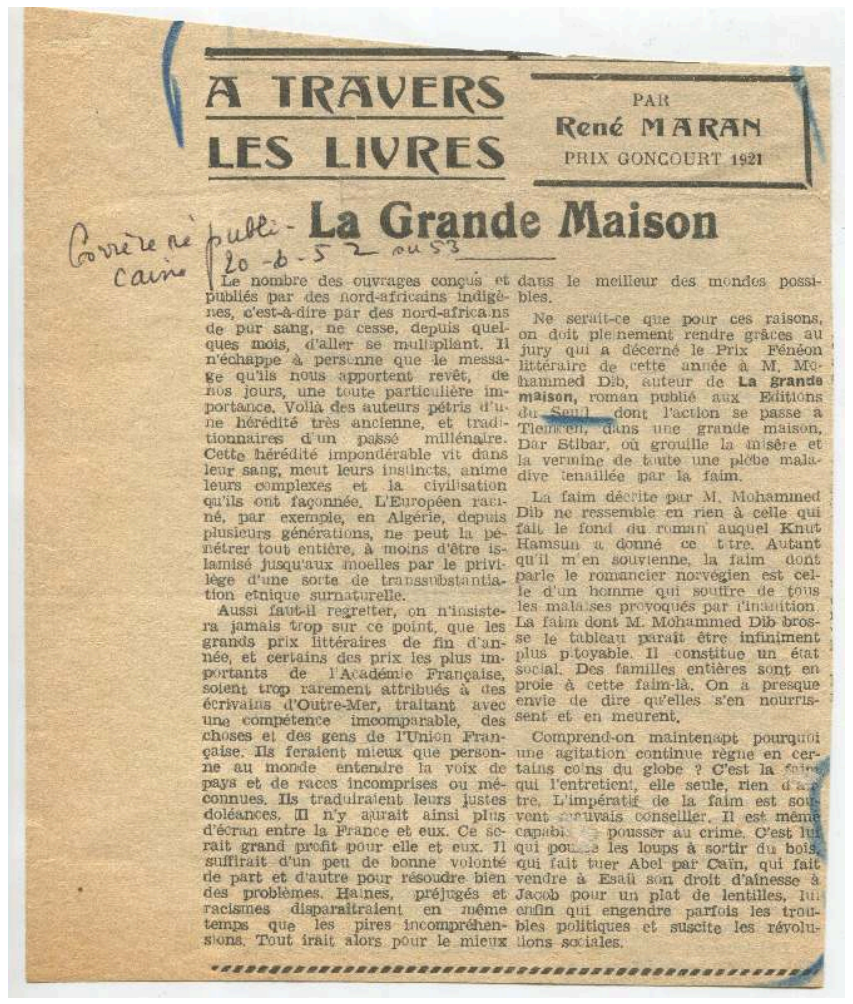

\section{Une distinction bien singulière}

Le premier élément frappant dans l'article critique de Maran réside dans la catégorisation ethnique appliquée par le Prix Goncourt 1921 au romancier algérien: après avoir employé l'expression «nord-africains indigènes ", les distinguant ainsi de leurs confrères d'origine européenne, Maran précise sa pensée, en usant d'une formulation, équivoque à première vue. Reconduisant la bipartition opératoire en colonie, le terme "nord-africains de pur sang » désigne donc les véritables autochtones du pays, qui selon l'écrivain antillo-guyanais, ont un "message " à délivrer, d'« une toute particulière importance ». Maran pointe alors les traditions " millénaires » dont ceux-ci sont porteurs, contrairement aux populations plus récemment implantées. Le terme "hérédité ", utilisé deux fois, semble attribuer à ceux qui en sont dotés des attitudes, un comportement spécifiques, se confondant avec leur sang même, des "instincts» auxquels ils ne peuvent se soustraire. Cependant, cette hérédité semble difficilement définissable, l'adjectif « impondérable » l'atteste. C'est cette conformation précise, propre aux autochtones de cette terre nord-africaine, qui échappe quelque peu aux Européens présents sur ce territoire, quand bien même leurs ancêtres s'y seraient implantés depuis plusieurs générations. Maran ne reconnaît pas de capacité de migration d'une communauté à une autre, sauf à de très rares exceptions, et au prix ajoute-t-il d'une « sorte de transsubstantiation ethnique surnaturelle »! 
4 Le terme "hérédité » est à mettre en relation avec cet autre terme employé ici par Maran, "instincts", terme que l'on retrouve dans son œuvre ainsi que dans sa correspondance $^{2}$ : il faut noter que ce terme est connoté de façon positive par Maran, et désigne sous sa plume cette morale utilitaire, puisée chez Rémy de Gourmont et son essai Physique de l'amour. Essai sur l'instinct sexuel, publié au Mercure de France en 1903, qui vise à assurer la reproduction et donc la conservation de l'espèce. Cet instinct sexuel et sa visée reproductive n'empêchent pas l'existence du plaisir, équivalent de la pulsion de vie. L'homme est donc libre, mais sa liberté s'exerce dans un cadre préétabli. Pour Maran, ainsi que De Gourmont, l'homme n'est pas supérieur aux autres êtres vivants dans la nature, mais un animal parmi d'autres ; la notion d'animalité n'est ainsi absolument pas perçue comme une notion dépréciative. La notion d'évolutionnisme, chère à Darwin, se voit remise en cause. En somme, l'instinct sexuel est associé à la lumière par Maran, et non la raison comme il est d'usage. Instinct qui bien que détaché a priori d'une perception moralisante, se confond sous la plume de Maran avec cette tyrannie de la chair à laquelle l'homme est soumis ${ }^{3}$.

\section{Quelle conception de la littérature?}

5 Maran accorde aux écrivains alors dits "indigènes " une valeur considérable, et déplore dans le deuxième paragraphe de son article le nombre insuffisant de prix attribués aux écrivains d'Outre-mer, ainsi qu'il les nomme. Ils sauraient se faire les porte-parole, les porte-voix de leurs "races incomprises ou méconnues ». Et Maran d'exhausser les œuvres de ces auteurs comme des cahiers de doléances ${ }^{4}$ adressés au lectorat de la métropole. «Il n'y aurait alors plus d'écran entre la France et eux. » Dib, pour sa part, concevait en des termes similaires le rôle de l'écrivain ; il affirme en 1958 dans un entretien publié dans Témoignage chrétien:

[...] il nous semble qu'un contrat nous lie à notre peuple. Nous pourrions nous intituler ses «écrivains publics». C'est vers lui que nous nous tournons d'abord. Nous cherchons à en saisir les structures et les situations particulières. Puis nous nous retournons vers le monde pour témoigner de cette particularité, mais aussi pour marquer combien cette particularité s'inscrit dans l'universel.

Néanmoins, la saisie par Mohammed Dib des structures et des situations relatives à son peuple l'amène à dénoncer l'iniquité du système colonial ; Maran, bien qu'il ait porté sur la place publique les injustices commises par la colonisation en Afrique noire, ne franchira jamais la ligne jaune et ne remettra pas fondamentalement en cause le bienfondé de l'œuvre coloniale française. Maran souhaite avant tout

qu'on supprime l'indigénat et sanctionne l'autoritarisme de l'administration coloniale. [...] l'anticolonialisme de [ce] (politiquement) modéré [le] conduit à stigmatiser les excès et les abus du système colonial et revendiquer du même coup une colonisation apte à assurer dans les colonies l'application des principes de la Révolution française ${ }^{5}$.

Dans l'une des premières séquences de LaGrande Maison, M. Hassan, l'instituteur d'Omar, avoue à demi-mot à ses jeunes élèves que la France n'est pas leur patrie, sans pour autant nommer pour l'heure leur véritable patrie. Il est vrai que trente ans séparent Batouala du roman de Mohammed Dib : les promesses inhérentes à l'entreprise coloniale n'ont pas été tenues et ont engendré au lendemain de la Seconde Guerre mondiale une rupture irréversible entre colonisateur et colonisé. 
C'est ainsi au nom d'une conception sociale de la littérature, et d'un rôle d'intermédiaire, de passeur entre l'identité française et son identité d'origine dévolu à l'écrivain d'Outre-mer, que Maran s'exprime ici. Cette fonction réaffirmée lui fit ainsi agréger en son temps à son célèbre premier roman, Batouala, véritable roman nègre ${ }^{6}$, une préface dénonçant les iniquités perpétrées en Afrique équatoriale française du temps qu'il y exerçait en tant qu'administrateur colonial. C'est cette aptitude à favoriser la communication, le dialogue entre des communautés diverses, que Maran entrevoit en la littérature, et qui lui fait espérer (selon un tour que d'aucuns peuvent juger candide) une échappatoire aux problèmes sociaux et politiques existant en colonie, ainsi qu'aux inimitiés dressant les communautés les unes contre les autres. Au nom de sa foi en l'universalisme de la nation française, d'un idéal de dialogue entre les peuples et de respect de l'altérité de chacun au sein du credo républicain et de l'Union française (en ce sens, sa fascination pour la figure de Savorgnan de Brazza s'explique aisément), Maran ne peut qu'être sensible au regard spécifique que les peuples colonisés possèdent et que leurs écrivains peuvent incarner dans leurs œuvres. Ainsi que Kusum Aggarwal le souligne au terme de son article à propos de ces deux personnalités :

[ê]tre Français, à leur sens, c'est agir en pleine conscience des possibilités que cela peut leur offrir d'étendre l'application parmi les peuples colonisés des idées républicaines et ainsi de participer dans le sillage de conquêtes révolutionnaires à l'établissement d'un empire républicain ${ }^{7}$.

\section{Une mise en garde qui interpelle}

7 Après avoir évoqué le prix Fénéon attribué au roman de Dib, Maran établit une comparaison entre le roman de l'écrivain norvégien Knut Hamsun, La Faim, et l'œuvre du romancier algérien. La Faim, publié en volume en 1890, met l'accent sur l'état physique et psychique du narrateur, causé par une faim abyssale qui ne cesse de provoquer la dégradation du personnage. La faim parcourant la fiction de Mohammed Dib revêt un caractère social ; elle finit par se superposer aux personnages eux-mêmes : celle que le jeune Omar nomme "Mère faim» finit par incarner l'état de sousdéveloppement endémique dans lequel est reléguée la population indigène. Cette faim contre laquelle Camus mettait en garde dans sa série d'articles de 1938-39, " Misère en Kabylie », est à prendre en considération selon René Maran; en une mise en garde formulée sans ambages, Maran pointe en conclusion de sa note de lecture les conséquences potentielles de cette faim persistante, à savoir « les troubles politiques » et autres "révolutions sociales" susceptibles d'éclater. Au-delà du facteur socioéconomique irriguant le roman, l'auteur de Batouala ignore la prise de conscience identitaire d'un peuple face à un occupant jugé illégitime et partant, sa volonté de recouvrer un Nom qui lui soit propre ${ }^{8}$ et de reprendre les rênes de sa destinée. Cependant, la note de lecture sur La Grande Maison apparaît hautement significative si le lecteur est en mesure de la replacer dans l'évolution de l'œuvre de Maran, et de ce que celle-ci révèle du positionnement de l'auteur vis-à-vis de l'entreprise coloniale. Entre la publication du second tome des Pionniers de l'Empire en 1946, et celle du troisième et dernier tome en 1955, l'adhésion pleine et entière à l'odyssée des précurseurs de la politique impériale de la France s'atténue, pour laisser place dans ce dernier tome au traitement de thèmes quelque peu dérangeants, et jusqu'ici passés sous silence par Maran : la dénonciation de la séparation des races par exemple, trop souvent règle commune en colonie, au profit de l'éloge d'un métissage dont l'auteur avait fait 
l'expérience dans son couple même; la dénonciation de la violence qui a trop souvent réglé les conquêtes de territoires au long des siècles; enfin, le doute exprimé sans détours quant à une pérennité de l'Empire français. Sylvie Brodziak qui analyse précisément cette évolution des positions maraniennes a pu ainsi écrire :

Seul le troisième tome, dont les quatre biographies parcourent le Sénégal et l'Asie au XVIII ${ }^{e}$ siècle, pose plus explicitement la question de la domination coloniale et constitue une rupture dans l'écriture jusqu'alors indulgente de la colonisation'.

Dans sa note de lecture, Maran n'entrevoit certes pas une révolution de type politique, et relève la seule faim comme motif d'insurrection, mais à moins de cinq mois du déclenchement de la Toussaint rouge, les derniers mots de Maran résonnent d'une portée toute prophétique. Les désagréments divers que lui ont valu sa conception humaniste de l'entreprise coloniale et la dénonciation des injustices commises alors qu'il était administrateur colonial trente ans plus tôt - dénonciations restées lettre morte - ne pouvaient que le rendre sensible au réquisitoire contre l'ordre colonial que constitue le roman de Dib.

9 Le lecteur pourrait alors percevoir la note de lecture de Maran comme un jalon emblématique de l'évolution politique de l'écrivain et le passage de témoin d'un précurseur à son cadet.

\section{NOTES}

1. René Maran, Un homme pareil aux autres, Paris, Arc-en-ciel, 1947, p. 25-26.

2. Je remercie Roseline Garcia Ballister qui a développé une réflexion le 12 mars 2021 dans le cadre du séminaire Maran de l'ITEM sur «Le fonctionnement de la conscience. La notion d'instinct chez Maran ». Ce terme d' « instinct » est également présent dans le roman Un homme pareil aux autres, paru aux éditions Arc en Ciel en 1947 : il y est question d'un " amour instinctif, profond, tout pétri de volupté ». Par ailleurs, dans une lettre adressée par Maran à son ami Manoël Gahisto du 11 janvier 1916, l'écrivain évoque «l'instinct de la conservation de la race, dont parlait Schopenhauer » (lettre inédite, je remercie Bernard Michel de m'avoir autorisé à la consulter).

3. Dans Un homme pareil aux autres $(1947$; 1962), il utilise l'expression "sacrifier à la bête " lorsqu'il évoque l'accouplement entre Jean Veneuse et Clarisse Nemours. Un hiatus entre l'idéalisation du sentiment amoureux et l'évocation du désir sexuel est clairement perceptible chez Maran.

4. Évoquant la littérature maghrébine de langue française des précurseurs, Abdellatif Laâbi, quelque quinze ans plus tard, déclarait dans le premier numéro de la revue Souffles, en son Prologue, ô combien célèbre : «On a l'impression aujourd'hui que cette littérature fut une espèce d'immense lettre ouverte à l'Occident, les cahiers maghrébins de doléances en quelque sorte. » (1966:3-6) L'appréciation, sous sa plume, revêt une charge critique teintée d'une certaine commisération.

5. Kusum Aggarwal, «René Maran : écrivain colonial ? Réflexions sur ses écrits biographiques d'après son Savorgan de Brazza (1951) », dans R. Little (dir.), René Maran : une conscience intranquille, Interculturel Francophonies, $\mathrm{n}^{\circ}$ 33, juin-juillet 2018, p. 238. 
6. René Maran, Batouala, véritable roman nègre, Paris, Albin Michel, 1921, réédité en 1938.

7. Ibid., p. 251.

8. Je fais ici allusion à l'argumentaire développé par Jean El Mouhouv Amrouche le 27 janvier1956 à la salle Wagram dans sa conférence «Guerre d'Algérie et colonialisme : quelques raisons du maquisard ", dans T. Yacine-Titouh (éd.), Un Algérien s'adresse aux Français. L'histoire d'Algérie par les textes (1943-1961), Paris, Awal/L'Harmattan, 1994, p. 28.

9. Sylvie Brodziak, « René Maran : l'écriture noire de la conquête blanche? », dans R. Little (dir.), René Maran : une conscience intranquille,op. cit., p. 266.

\section{RÉSUMÉS}

René Maran et Mohammed Dib ne se sont jamais rencontrés. Une génération les sépare, outre leur appartenance à un champ géographique différent. Maran a pourtant consacré une note de lecture fort intéressante au premier roman de Mohammed Dib. Sa recension consacrée à La Grande Maison, roman paru au printemps 1952, publiée dans La Corrèze républicaine le 20 juin 1952 lui a permis de formuler certains paramètres inhérents à sa vision idéologique et politique.

René Maran and Mohammed Dib have never met. A generation separates them, apart from their belonging to a different geographical field. Maran has nevertheless devoted a very interesting reading note to Mohammed Dib's first novel. His review of La Grande Maison, a novel published in the spring of 1952 and published in La Corrèze républicaine on 20 June 1952, allowed him to formulate certain parameters inherent to his ideological and political vision.

\section{INDEX}

Keywords : René Maran, Mohammed Dib, Algeria, La Grande Maison Index géographique : Algérie

Mots-clés : René Maran, Mohammed Dib, Algérie, La Grande Maison

\section{AUTEUR \\ HERVÉ SANSON \\ ITEM/CNRS}

